\title{
Study Population Description
}

National Cancer Institute

\section{Source}

National Cancer Institute. Study Population Description. NCI Thesaurus. Code C70834.

A textual description of the target study population which contains precisely defined characteristics of potential participants. This includes inclusion and exclusion criteria, the number of potential participants, conditions of recruitment and sample structure, information on vulnerable population(s) involvement in the study (if applicable). Other available appropriate characteristics of the population from which study subjects will be recruited and to which the study results could be reasonably generalized is considered noteworthy. 